\title{
Optical properties of photonic crystals composed of metal-coated spheres
}

\author{
Jensen $\mathrm{Li}^{1}{ }^{1}$ Gang Sun, ${ }^{1,2}$ and C. T. Chan ${ }^{1}$ \\ ${ }^{1}$ Department of Physics, The Hong Kong University of Science and Technology, Clear Water Bay, Kowloon, Hong Kong, China \\ ${ }^{2}$ Institute of Physics, Chinese Academy of Sciences, Beijing, China
}

(Received 27 September 2005; published 23 February 2006)

\begin{abstract}
We consider the optical properties of photonic crystals composed of metal-coated dielectric spheres. The calculations are performed using the multiple-scattering theory as well as a model of coupled dipole resonators that gives an intuitive understanding of band dispersions. The low frequency dispersions are found to originate from the hybridization of the dipolar resonance with the free-space propagating electromagnetic modes. We derived a wave-vector dependent effective-medium theory that can describe the low frequency resonating behavior very well. Within this new formulation, the effective constitutive parameters become $k$-dependent. In contrast to the standard Maxwell-Garnett type effective medium theory, which gives a gap near the resonances, the wave-vector dependent effective-medium theory can give the dispersion of the resonance bands. The very robust directional band gap between the second and the third band in the face-centered-cubic metallo-dielectric photonic crystals is found to originate from the dipole resonance of the coated spheres. The directional gap of the simple cubic structure also originates from the dipole resonance and the coupling to higher order resonances leads to an absolute gap.
\end{abstract}

DOI: 10.1103/PhysRevB.73.075117

PACS number(s): 78.20.Ci, 73.20.Mf, 42.70.Qs, 78.67.Bf

\section{INTRODUCTION}

Metallo-dielectric photonic band gap systems, composed of metal spheres or metal-coated spheres embedded in a dielectric host, have attracted some attention. ${ }^{1-5}$ These systems are found to exhibit rather robust photonic band gaps, strongly modified dispersion surfaces and density of states. While the photonic band gaps of conventional dielectric photonic crystals are the consequences of Bragg scattering, the gaps of metallo-dielectric photonic crystals can result from resonances of the individual spheres as well. We will trace the origin of the lowest frequency band gap and show that the lowest photonic band gap is derived from dipolar resonance. In order to give a more comprehensive picture, we have considered coated spheres with varying thicknesses of metal coating. The resonance frequencies, and hence the photonic band gap and the dispersions, can be tuned by varying the thickness of the coating, and the overall size of the spheres. We note that there are significant advances in the synthesis of metal-coated dielectric particles or hollow metallic nano-shells in recent years ${ }^{6-9}$ and the optical properties of both a single nano-shell or a nano-shell cluster are also investigated experimentally and theoretically. ${ }^{10-20}$

In addition to the fully fledged multiple-scattering and the coupled resonator approach, we also formulated a new $k$-dependent effective medium theory, which gives a good description of the optical properties of such systems in the low frequency regime. This extended effective medium formulation may be useful in other applications, and details of the mathematics are given in the Appendices.

\section{METHODS OF CALCULATION}

In this paper, we will use different methods to consider the optical properties of a crystal of metal-coated microspheres. These methods are namely (i) the effective medium
Maxwell-Garnett (MG) method, (ii) $k$-dependent effective medium method, and (iii) multiple-scattering method. The purpose of using a hierarchy of methods is to expose the physics of the problem at different levels of complexity. Since the MG theory is well-known, and the mathematical details of the multiple-scattering method have been documented in the literature, ${ }^{21}$ they will not be discussed here except by noting that the multiple-scattering theory gives an essentially exact solution. The $k$-dependent effective medium theory can be summarized in Eqs. (5) and (7), and the mathematical details that lead to these equations are given in Appendix $\mathrm{B}$. We will see that the $k$-dependent effective medium theory reduces to the standard MG theory near the Brillouin zone center and is equivalent to a multiple-scattering method with maximum angular momentum index of $L_{\max }=1$ in the low frequency limit, so it bridges the two formulations.

\section{EFFECTIVE MEDIUM RESULTS}

We first note that the limitations of Maxwell-Garnett type effective medium in the context of the optical properties of plasmon spheres have been examined previously. ${ }^{22,23}$ In this section, we apply the MG theory to obtain the optical properties of metal-coated spheres and then we consider its extension.

Here, we examine a colloidal crystal of Ag-coated glass spheres. The optical properties of small metallic particles embedded in a dielectric host are quite well known. This type of composite material, in the low frequency regime where the wavelength in the dielectric background is much larger than the average inter-particle distance " $\alpha$," is well modeled by the effective medium theory in which the composite is replaced by a homogeneous material with an effective permittivity $\epsilon_{\text {eff }}$. In the lowest order dipole approximation (valid for small volume filling ratio $f$ ), $\epsilon_{\text {eff }}$ can be found by the Maxwell-Garnett (MG) formula, ${ }^{24}$ which states that 




FIG. 1. Dispersion of an FCC structure of Ag-coated glass spheres $\left(r_{\text {glass }}=84 \mathrm{~nm}, r_{\mathrm{Ag}}=88 \mathrm{~nm}\right)$ with a volume filling ratio $f$ $=0.2584$ from the Maxwell-Garnett formula. We choose to label the $k$-vectors with that of a FCC Brillouin zone for easier comparison with subsequent figures.

$$
\frac{\epsilon_{\mathrm{eff}}-\epsilon_{m}}{\epsilon_{\mathrm{eff}}+2 \epsilon_{m}}=f \frac{\epsilon_{s}-\epsilon_{m}}{\epsilon_{s}+2 \epsilon_{m}},
$$

where $\epsilon_{m}\left(\epsilon_{s}\right)$ is the permittivity of the dielectric background (particle) relative to vacuum.

If $\epsilon_{s}$ is negative, as in the case of metals, $\epsilon_{\text {eff }}$ will have resonating behavior. It implies that the composite material will have poor transmission and strong absorption. Within the context of MG theory, there is a plasmon absorption band (a peak when we measure the attenuation coefficient of the material) in the frequency range when the MG formula gives negative values of $\epsilon_{\text {eff }}$ ranging from minus infinity to zero. It in turns requires a resonance in the right-hand-side of the MG formula, which is proportional to the polarizability of a single particle. When the filling ratio approaches zero, the frequency range collapses to a single frequency $\omega_{\mathrm{F}}$, which denotes the Fröhlich surface mode ${ }^{25}$ of a single metallic particle, given by

$$
\epsilon_{s}\left(\omega_{\mathrm{F}}\right)=-2 \epsilon_{m} .
$$

Therefore, the material can be engineered through modifying the $\omega_{\mathrm{F}}$. Here, we employ metal-coated dielectric particles which can support a Fröhlich surface mode at an even lower frequency than the solid metallic particle.

As an example, Fig. 1 shows the dispersion of a colloidal crystal of spherical Ag-coated glass particles with the radius of the glass core being $84 \mathrm{~nm}$ and the radius of the outer surface of the Ag coating being $88 \mathrm{~nm}$ using the MG formula in the form

$$
\frac{\kappa^{2}-k_{0}^{2}}{\kappa^{2}+2 k_{0}^{2}}=f \frac{\epsilon_{s}-1}{\epsilon_{s}+2},
$$

where $\kappa=k_{0} \sqrt{\epsilon_{\mathrm{eff}}}, k_{0}=\omega / c$, and $c$ is the speed of light in vacuum. The dielectric function of $\mathrm{Ag}$ is taken from experimental values. ${ }^{26}$ Since we want to study the dispersion of such a system, we will just take the real part of the dielectric function of $\mathrm{Ag}$ for the moment. The issue of absorption will be considered in later parts of the paper. As the MG formula depends only on the filling ratio, there is no need to specify the arrangement of the spheres. But for more straightforward comparison with the results in the following sections, we will label the $k$-space with that of the Brillouin zone of a FCC lattice in Fig. 1. In the low frequency regime where only the dipolar response of the particle is important, the coated particle is effectively equivalent to a homogeneous particle with an effective permittivity $\left(\epsilon_{s}\right)$ of a chosen radius $\left(r_{s}\right.$, which is usually taken as the actual radius of the particle). Details are given in Appendix A. The $\epsilon_{s}$ [to be substituted into Eq. (3)] is governed by

$$
\frac{\epsilon_{s}+2}{\epsilon_{s}-1}=4 \pi r_{s}^{3}\left(\frac{1}{\alpha}+\frac{i k_{0}^{3}}{6 \pi}\right),
$$

where $\epsilon_{0} \alpha$ is defined as the electric polarizability of the coated particle in vacuum and $\epsilon_{0}$ is the permittivity of vacuum. From the dispersion, we see that a band gap appears from about 1.1 to $1.4 \mathrm{eV}$. It is the plasmon absorption "band" (which perhaps should be called the plasmon gap) centered at the resonance due to the Fröhlich surface mode. In fact, for the coated particle, there are exactly two frequencies in which the condition of Fröhlich surface mode [Eq. (2)] is satisfied where $\epsilon_{s}$ now means the effective permittivity of the whole particle with coating. It is the result of the coupling between the sphere plasmon on the outer surface and the cavity plasmon on the inner surface of the metal. The one having a smaller frequency is the sphere-like mode while the one having a higher frequency is the cavity-like mode. ${ }^{17,18}$ From now on, the Fröhlich surface mode will mean the sphere-like mode as the cavity-like mode is at a frequency high enough that the effective medium approximation is not appropriate and it is also too sensitive to the absorption of metal in the systems we are considering. We note that there is a dispersion-less (flat) band at $1.4 \mathrm{eV}$. It is the nondegenerate longitudinal mode supported at the frequency where the effective permittivity is zero. All the other bands are doubly degenerate transverse modes due to the assumed effective medium. The dispersion-less longitudinal band meets at the bottom of the parabolic band since there is no way to distinguish a transverse and a longitudinal mode exactly at the $\Gamma$ point in the Brillouin Zone.

We note that the MG formula is only valid when the induced dipole profile varies slowly from particle to particle ( $\kappa a \ll 1$ with $a$ being the lattice constant). It means that if we have an ordered crystalline array of spheres, in which case we can talk about a band structure, MG formula is valid only near the zone center. On the other hand, when the particles are assembled into an ordered lattice, we can treat it as a photonic crystal and its optical properties can be calculated using the multiple-scattering method. ${ }^{22}$ In this way, propagating solutions far from the Brillouin zone center can also be obtained accurately and the effect of higher multipoles can also be included.

The dispersion calculated by the multiple-scattering method is shown as open circles in Fig. 2. The coated spheres are arranged into an FCC structure, with a volume filling ratio of $f=0.2584$ in Fig. 2(a) and $f=0.15$ in Fig. 2(b). Figures 1 and 2(a) can be compared directly as they have the same filling ratio. The multiple-scattering method expands the fields using a linear combination of vector spherical har- 


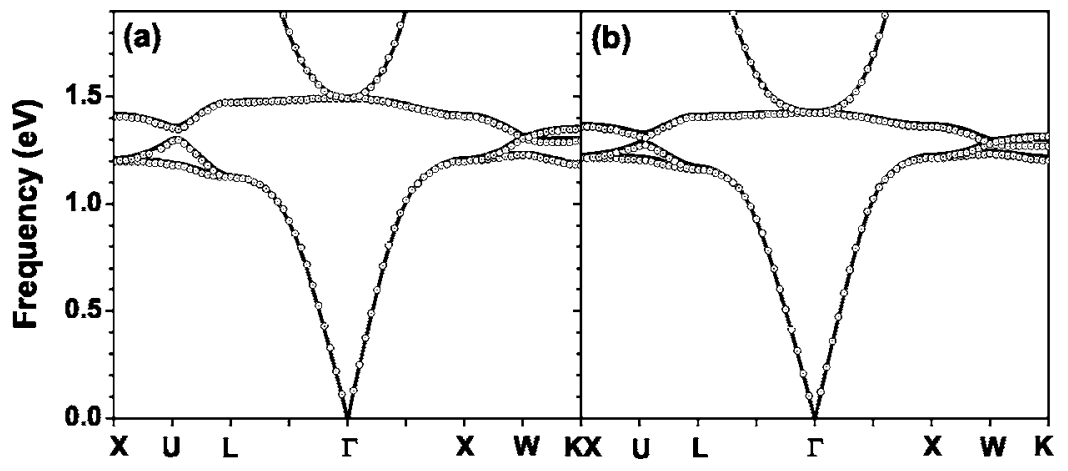

FIG. 2. Dispersion of an FCC structure of Agcoated glass spheres $\left(r_{\text {glass }}=84 \mathrm{~nm}, r_{\mathrm{Ag}}=88 \mathrm{~nm}\right)$ with a volume filling ratio $f$ of (a) 0.2584 and (b) 0.15 , respectively. The open circles show the multiple-scattering results up to the dipole approximation $\left(L_{\max }=1\right)$. The lines show the dispersion from the extended Maxwell-Garnett formula in Eq. (7).

monics, and for the moment, we restrict the expansion to an angular momentum index of $L_{\max }=1$ (i.e., dipole contributions only). In fact, the contribution from the higher multipoles is very small in the low frequency regime that we are focusing on. By comparing Figs. 1 and 2(a), we see that near the zone center where the dipoles are slowly varying from particle to particle, the MG formula is a good approximation except for a small numerical discrepancy due to a dynamicstructure-correction term on the near field (see Appendix B for the details). With the MG formulation, the particles outside the Lorentz sphere are treated as a continuum while the contribution from particles within the Lorentz sphere is taken as zero, which is assumed to be correct for a random (uncorrelated) distribution, and is true for a cubic lattice at the quasi-static limit.

The comparison of Figs. 1 and 2(a) shows that MG results capture some salient features of the multiple-scattering theory, except that the forbidden frequency range in $\mathrm{MG}$ theory (from about 1.1 to $1.4 \mathrm{eV}$ ) becomes a set of 3 bands in the multiple-scattering result, which are flat near the zone center, and becomes dispersive at the zone boundary. Although the multiple-scattering result shown in Fig. 2(a) is restricted to the dipole order, there is still a difference between the MG and the multiple-scattering result because the MG theory is essentially a continuum model with no structural information, while multiple-scattering naturally builds in structural information. By comparing Figs. 2(a) and 1, we can attribute the rather flat bands near $1.4 \mathrm{eV}$ to the resonance due to the coating of the individual spheres. In Fig. 2(b), we show the multiple-scattering calculated dispersion for the same spheres, but at a lower filling ratio of $f=0.15$ (at an expanded lattice constant). We see that the features are very nearly the same as that in Fig. 2(a), in particular that the flat bands are at about the same frequency, except that the bandwidth is smaller. This is expected from a resonancedriven mechanism. We note that if the dispersion originates from Bragg scattering, it will depend on the lattice constant and thus the filling ratio.

Far from the zone center, the photonic band structure found from the multiple-scattering method shows that the first two transverse bands are rather dispersive instead of becoming flat. They are split near the U point. The gap becomes smaller in size in general in the region far from the zone center and it closes at the $\mathrm{W}$ point. In the original dispersion from the MG formula, the first doubly transverse band becomes flat far away from the $\Gamma$ point, representing an effective permittivity of negative infinity. The MG formula does not work far from the $\Gamma$ point. In other words, it is not expected to work in the current case because the dispersion is not linear anymore. The surface plasmon resonance of the Ag coating causes resonance bands which cannot be described by the MG theory. We will now attempt to extend the MG formula so that it can cover the full Brillouin zone.

By solving the Bloch eigenstates under the dipole approximation in the low frequency regime, it can be proved (see Appendix B) that we can define a wave vectordependent effective permittivity tensor $\overleftrightarrow{\epsilon}_{\text {eff }}(\boldsymbol{\kappa})($ a $3 \times 3$ matrix) by

$$
\frac{\overleftrightarrow{\epsilon}_{\mathrm{eff}}(\boldsymbol{\kappa})+2 \stackrel{\leftrightarrow}{I}}{\overleftrightarrow{\epsilon}_{\mathrm{eff}}(\boldsymbol{\kappa})-\stackrel{\leftrightarrow}{I}}+3 F\left[\frac{3 \hat{r} \hat{r}-\stackrel{\leftrightarrow}{I}}{4 \pi r^{3}}\right]+3 k_{0}^{2} F\left[\frac{\stackrel{\leftrightarrow}{I}+\hat{r} \hat{r}}{8 \pi r}\right]=\frac{1}{f} \frac{\epsilon_{s}+2}{\epsilon_{s}-1} \stackrel{\leftrightarrow}{I}
$$

where the transform $F$ is defined by

$$
F[f(\mathbf{r})](\boldsymbol{\kappa})=\lim _{R_{0} \rightarrow \infty} \Omega_{a} \sum_{0<|\mathbf{R}|<R_{0}} f(\mathbf{R}) e^{i \boldsymbol{\kappa} \cdot \mathbf{R}}-\int_{0}^{R_{0}} d r^{3} f(\mathbf{r}) e^{i \boldsymbol{\kappa} \cdot \mathbf{r}}
$$

In the above two formulas, $\stackrel{\leftrightarrow}{I}$ is the identity tensor (a $3 \times 3$ matrix), $\mathbf{R}$ is any Bravais lattice vector and $\Omega_{a}$ is the volume of a primitive unit cell. Note that the second term on the left hand side of Eq. (5) becomes zero for the local effective medium $(\boldsymbol{\kappa} \longrightarrow \mathbf{0})$ while the third term becomes zero at the quasi-static limit. Therefore, Eq. (5) returns to the MG formula if we are considering the local effective medium at the quasi-static limit. Now, the dispersion curve can be obtained from solving the Maxwell equations for a homogeneous medium with permittivity $\overleftrightarrow{\epsilon}_{\text {eff }}(\boldsymbol{\kappa})$ and $\nabla \rightarrow i \boldsymbol{\kappa}$ while the permeability is set to one as the magnetic response for small metallic particles can be neglected. For any cubic lattices (FCC, $\mathrm{BCC}$, or SC), it can be further approximated (see Appendix B) by

$$
\frac{1}{3} \frac{\kappa^{2}-k_{0}^{2}}{k_{0}^{2} t_{i}(\boldsymbol{\kappa})+\left(\kappa^{2}-k_{0}^{2}\right)\left(\lambda_{i}(\boldsymbol{\kappa})+k_{0}^{2} \tau_{i}(\boldsymbol{\kappa})\right)}=f \frac{\epsilon_{s}-1}{\epsilon_{s}+2},
$$

where

$$
\begin{gathered}
\overleftrightarrow{U}_{s}(\boldsymbol{\kappa}) \cdot \hat{P}_{i}=\lambda_{i}(\boldsymbol{\kappa}) \hat{P}_{i}, \\
t_{i}(\boldsymbol{\kappa})=\hat{P}_{i} \cdot(\stackrel{\leftrightarrow}{I}-\hat{\kappa} \hat{\kappa}) \cdot \hat{P}_{i},
\end{gathered}
$$


TABLE I. Functional values of $\lambda_{i}(\boldsymbol{\kappa}), t_{i}(\boldsymbol{\kappa}), \tau_{i}(\boldsymbol{\kappa})$ at the various high-symmetry points for the FCC structure.

\begin{tabular}{cclc}
\hline \hline & $\lambda_{i}$ & \multicolumn{1}{c}{$t_{i}$} & $\tau_{i}$ \\
\hline$\Gamma$ & +0.33333 & 1 & -2.40030 \\
& +0.33333 & 1 & -2.40030 \\
& -0.66667 & 0 & -2.40030 \\
& +0.17245 & 1 & -1.81106 \\
& +0.17245 & 1 & -1.81106 \\
$W$ & -0.34490 & 0 & -1.91869 \\
& +0.12542 & 0.80008 & -1.62217 \\
& -0.06269 & 1 & -2.12630 \\
$K$ & -0.06273 & 0.19992 & -1.48624 \\
& +0.21327 & 1 & -1.64099 \\
& -0.03312 & 0 & -1.25853 \\
& -0.18015 & 1 & -2.43184 \\
& +0.21327 & 1 & -1.64099 \\
& -0.03308 & 0.88895 & -2.04870 \\
& -0.18019 & 0.11105 & -1.64167 \\
& +0.28769 & 1 & -1.56558 \\
& +0.28769 & 1 & -1.56558 \\
& -0.57539 & 0 & -2.27959 \\
\hline \hline
\end{tabular}

$$
\tau_{i}(\boldsymbol{\kappa})=\hat{P}_{i} \cdot F\left[\frac{\overleftrightarrow{I}+\hat{r} \hat{r}}{8 \pi r}\right] \cdot \hat{P}_{i}
$$

$\lambda_{i}$ and $t_{i}(i=1,2,3)$ are the eigenvalues and the "transverse factor" ranging from 0 (longitudinal) to 1 (transverse) for the three eigenmodes of the Fourier transformed static dipole propagator $\overleftrightarrow{U}_{s}(\boldsymbol{\kappa})=\Omega_{a} \Sigma_{R>0}\left[(3 \hat{R} \hat{R}-\stackrel{\leftrightarrow}{I}) / 4 \pi R^{3}\right] e^{i \boldsymbol{\kappa} \cdot \mathbf{R}}$. The term $k_{0}^{2} \tau_{i}(\boldsymbol{\kappa})$ is called the dynamic-structure-correction term here. All these quantities are purely real for cubic crystals and the eigenvectors $\hat{P}_{i}$ are normalized to have unit magnitude.

From Appendix B, we see that Eq. (7) differs from the exact $L_{\max }=1$ multiple-scattering formulation in the sense that it assumes the existence of a Lorentz sphere within which we can expand the exact Green function by Taylor series in frequency. Both are good in the low frequency regime. The frequency dependent single particle property only appears on the right hand side of Eq. (7) such that the Bloch state eigenvalue problem is reduced to an eigenvalue problem which is structure dependent only. The eigenvalue problem of the static dipole propagator ${ }^{27}$ from Eq. (8) can be solved in the whole first Brillouin zone beforehand to get $\lambda_{i}(\boldsymbol{\kappa}), t_{i}(\boldsymbol{\kappa})$, and $\tau_{i}(\boldsymbol{\kappa})$ which are now regarded as some kinds of special functions of the wave vector. Their values at the high-symmetry points are listed in Table I. Comparing with the MG formula in Eq. (3), we see that Eq. (7) has a similar mathematical form, but its left hand side has the structure dependence and it is now valid in the whole Brillouin zone. The band structures obtained from Eq. (7) is shown as a solid line in Fig. 2. We see that it agrees very well with the actual band structure calculated from the multiple-scattering method. Equation (7) can reproduce both the transverse and



FIG. 3. Dispersion of an SC structure of Ag-coated glass spheres $\left(r_{\text {glass }}=84 \mathrm{~nm}, r_{\mathrm{Ag}}=88 \mathrm{~nm}\right)$ with a filling ratio of 0.2584 . The open circles show the multiple-scattering result up to $L_{\max }=1$. The lines show the dispersion calculated using the extended Maxwell Garnett formula in Eq. (7).

the longitudinal bands. When the Bloch wave vector approaches the zone center, $\overleftrightarrow{U}(\boldsymbol{\kappa}) \rightarrow \stackrel{\leftrightarrow}{I} / 3-\hat{\kappa} \hat{\kappa}$, both $3 \lambda_{i}$ and $t_{i}$ approaches the value one independent of lattice structure for the doubly degenerate transverse band, and Eq. (7) returns to the MG formula if we neglect the dynamic-structurecorrection term. While the MG formula gives the same results for different structures (as long as filling ratio is the same), and is not valid if the dispersion is not linear; Eq. (7) gives the correct structural dependence and can describe correctly the dispersion when there are resonances, in which case the dispersion cannot be linear.

When the same kind of Ag-coated glass particles with the same radii are assembled into a simple cubic (SC) structure instead of an FCC structure with the same filling ratio, the photonic band structure calculated by the multiple-scattering method (with $L_{\max }=1$ ) is compared with the extended MG formula in Fig. 3. The extended Maxwell Garnett formula predicts the exact band structure very well and also the directional gap due to plasmon resonance.

For different cubic lattices, we found that there are degeneracies at the zone boundary. These three bands for different cubic lattice meet at some point near the Brillouin zone edge. For example, the bands cross at the $\mathrm{W}$ point for $\mathrm{FCC}$ and the $\mathrm{R}$ point for $\mathrm{SC}$ structure. Therefore, from the band structure in the dipole approximation, the resonance gap is not an absolute gap. However, it is an omnidirectional gap since it is a gap for propagating wave of any incidence angle. Coupling to higher multipoles may open an absolute gap.

When the wave vector is far from the Brillouin zone center $\left(\kappa \gg k_{0}\right)$, the interaction between the single particle resonance and the light line can be neglected. We take the approximation that the speed of light approaches infinity (without retardation), the dipolar band structure from Eq. (7) can be approximated by the eigenvalues of the static dipole propagator as

$$
\frac{1}{\lambda_{i}(\boldsymbol{\kappa})}=3 f \frac{\epsilon_{s}-1}{\epsilon_{s}+2} .
$$

Figure 4 shows the dispersion of the FCC structure using Eq. (9). The dispersion of these bands can be understood from 


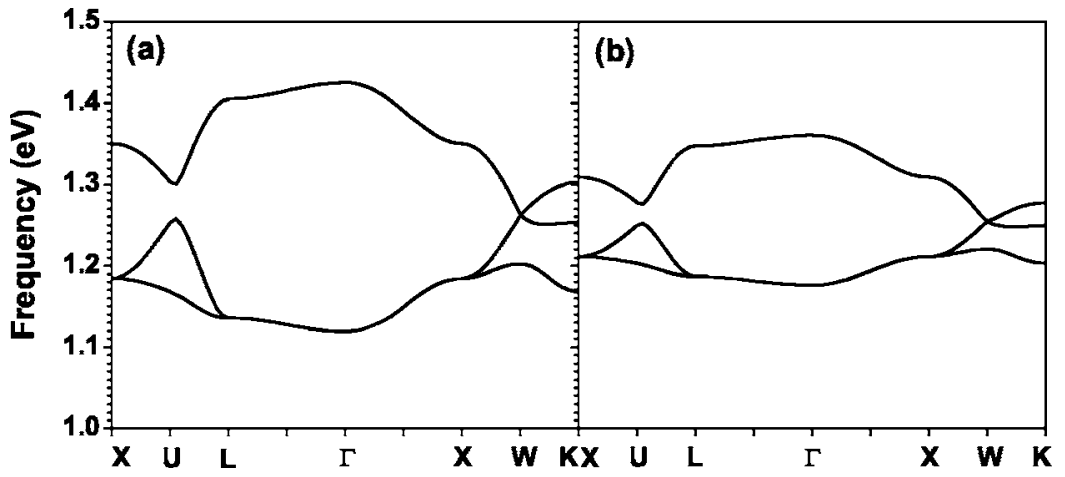

FIG. 4. The dispersion of an FCC structure of Ag-coated glass spheres $\left(r_{\text {glass }}=84 \mathrm{~nm}, \quad r_{\mathrm{Ag}}\right.$ $=88 \mathrm{~nm}$ ) according to Eq. (9) with a filling ratio of (a) 0.2584 , and (b) 0.15 , respectively. This dispersion can be regarded as the bands formed out of the resonance of the individual spheres. A higher filling ratio gives a wider band width. Near the zone center, the upper band is a longitudinal mode, and the lower band is a twofold degenerate transverse mode. Hybridization of these resonance bands with the linear dispersion of the vacuum gives the band structure in Fig. 2. the point of view of a tight binding picture that the Fröhlich surface mode of single particles in the lattice couple together to form the bands. The coupling is taken to be without retardation in this picture because we neglect the interaction between the Fröhlich surface mode and the light line. By comparing Figs. 4 and 2, Eq. (9) gives the salient feature of the band structure in the region far from the Brillouin zone center. The lower two bands are transverse and the upper band is longitudinal in nature. In the hybridization picture, the two transverse bands in Fig. 4 hybridize with the vacuum states (light line) to give the dispersion in Fig. 2. We should note that within a MG description, a resonance opens an absolute gap. However, it is actually not true in a more accurate description. The lowest three bands in an FCC structure are the result of the coupling between the "tight-binding" bands shown in Fig. 4 with the free space EM mode. We see that the resonance ensures a gap near the zone center. However, the tight-binding bands derived from the dipolar resonance actually have no gap at some zone boundary points (due to the particular symmetry of the lattice). The resonance thus opens up omni-directional gaps, but not absolute gaps.

\section{PHOTONIC BAND GAP CALCULATION BY THE MULTIPLE-SCATTERING APPROACH}

For pure Ag particles in air, the photonic band gap created by the Fröhlich surface mode is approximately at $3.5 \mathrm{eV}$ (wavelength in vacuum around $350 \mathrm{~nm}$ ). It is usually far beyond the low frequency regime so that an effective medium description of the material is not accurate unless the spacing of the particles is very small (say less than $30 \mathrm{~nm}$ ). However, from the last section, the Fröhlich frequency of the particle can be shifted downwards from the one of a pure Ag particle $(\sim 3.5 \mathrm{eV})$ to the one of an Ag-coated glass particle $(\sim 1.25 \mathrm{eV})$. When the coating is thin enough, the Fröhlich frequency can be pushed to a very low frequency so that effective medium theory becomes quantitatively correct. Therefore, by varying the coating thickness we can tune the gap frequency. In fact, this photonic band gap only depends on the resonating behavior of a single particle. The frequency of the resonance gap will not shift with the lattice constant in contrary to photonic band gaps that are derived from Bragg scattering.

In the last section, within the dipole approximation, we know that a resonance on the right hand side of Eq. (7) opens a gap in the photonic band structure. In fact, it can be ex- tended to the intermediate frequency regime (beyond the low frequency regime) that a dipolar resonance of the single particle is responsible for the opening of a photonic band gap at that particular frequency, although the effective medium description losses its accuracy due to the influence of the higher multipoles. Equation (7) can still be used to calculate the band structure qualitatively. Note that $\epsilon_{s}$ on the right hand side of Eq. (7) remains real even if we move away from the low frequency regime. This can be proved from Eq. (4) by considering the absorption power of a nonabsorbing particle.

To examine this resonance photonic gap in detail, the transmission and reflection spectra for normal incidence on the (111) surface of a photonic crystal in an FCC structure consisting of Ag-coated spherical glass particles at a fixed filling ratio of 0.577 is shown in Fig. 5. The frequency is given in normalized frequency units defined as $\omega \alpha / c$ where $\alpha$ (fixed at $250 \mathrm{~nm}$ ) is the sphere-to-sphere distance on the (111) surface and $c$ is the speed of light in vacuum. The radius of the glass core is varied so that the coated sphere can change from a pure glass particle to a pure Ag particle, while the outer radius of the coated particle is fixed at $r_{\mathrm{Ag}}$ $=115 \mathrm{~nm}(0.46 \alpha)$. The results are calculated by the multiplescattering method (with high enough multipole terms, up to an angular momentum index of 19 , to be included to guarantee full convergence). For the case of a very thin coating $\left(r_{\text {glass }}=0.455 \alpha\right)$, the dispersion agrees very well with the band structure for the first photonic band gap obtained from the dipole approximation in Eq. (7). In the thin coating limit, the physics can thus be fully accounted for by the dipole model. When the coating thickness increases, this photonic gap shifts progressively to higher frequencies into the intermediate frequency regime. In particular, we have plotted the exact band structure of the case $r_{\text {glass }}=0.44 \alpha$ in Fig. 6 and the same band structure only up to the dipole approximation in Fig. 7 as well. At the intermediate frequency, the salient features in the lowest three bands can still be explained by the dipole approximation. When we plot the extinction crosssection efficiency of a single sphere, we see that the first peak (indicating the dipolar resonance) in fact coincides with the gap. It verifies that the gap in the band structure is due to dipolar resonance (not due to Bragg scattering). In order to predict the gap position accurately, we should use the polarizability of a finite-size sphere instead of the one at quasistatic limit (which corresponds to a point dipole). The higher multipole terms only shift the band position below and above the gap in the intermediate frequency regime while they almost have no effects on the bands at low frequencies. From 


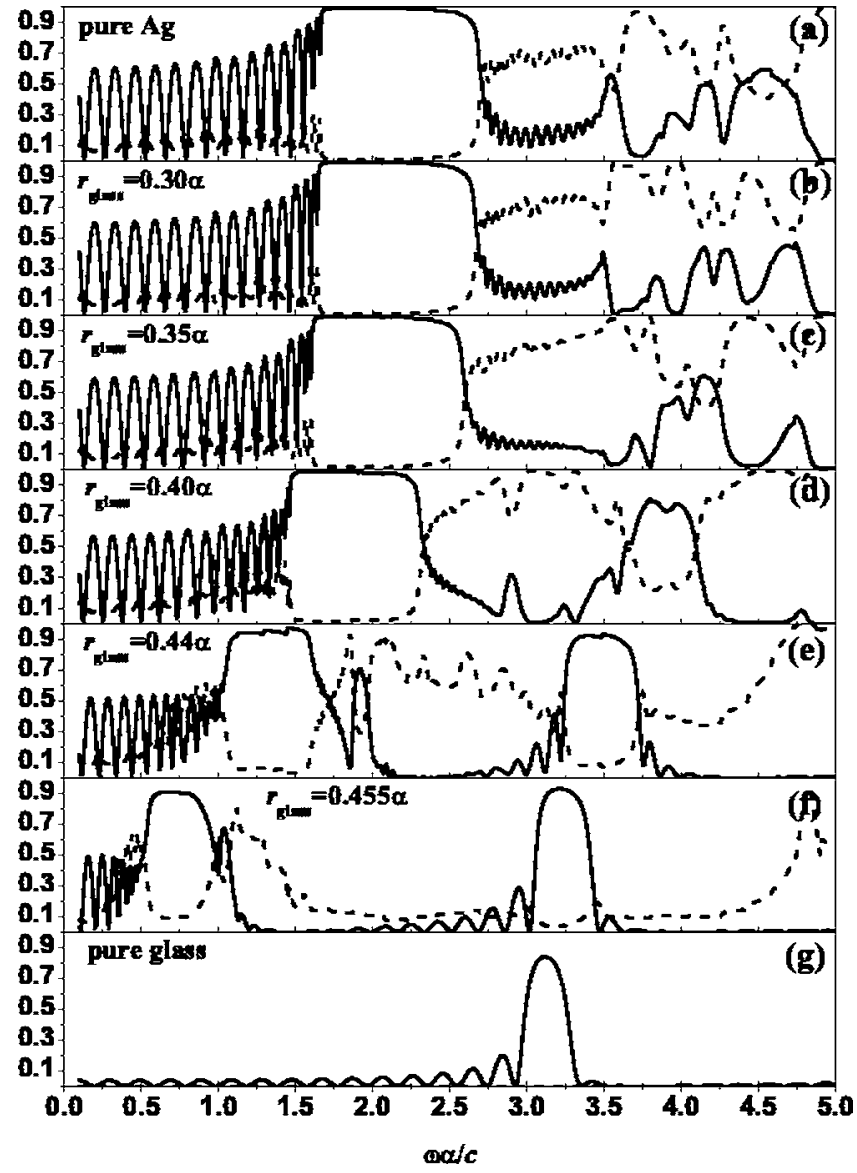

FIG. 5. Reflection spectrum (solid line) for normal incidence on 16 layers of an FCC (111) surface consisting of Ag-coated glass spherical particles at a volume filling ratio of 0.577 calculated by the multiple-scattering method. The outer radius of the coated particle is fixed at $115 \mathrm{~nm}(0.46 \alpha)$. The dashed lines show the corresponding absorbance.

the band structure, we see there are a bundle of bands lying above the first gap. These flat bands come from the resonances of higher multipoles. At even higher frequencies, the band structure returns to the one of pure glass sphere. In that frequency regime, $\mathrm{Ag}$ has a large skin depth so that the incident wave can probe the glass core. The whole particle behaves like a dielectric sphere very similar to glass. Thus, the directional gap (also shown in the transmission spectrum) in that frequency regime is in fact due to the Bragg scattering of the glass spheres. We note that Eq. (7) can only predict the resonance gap in the band structure but not the Bragg gap. Moreover, while Eq. (7) predicts the position of the dipolar resonance gap, it neglects the higher multipolar response of the system. The higer multipolar bands usually lie above the dipolar resonance gap but we also note that for systems of higher filling ratio (especially near the touching-sphere limit and Fröhlich surface mode of further lower frequency), the dipolar approximation becomes inappropriate and the multipolar bands can even lie inside the dipolar resonance gap. ${ }^{23}$

As we further decrease the radius of the glass core until the whole sphere becomes pure Ag, we found that the extinction cross section efficiency of a single sphere still has a peak due to the dipolar term centered at the first photonic band

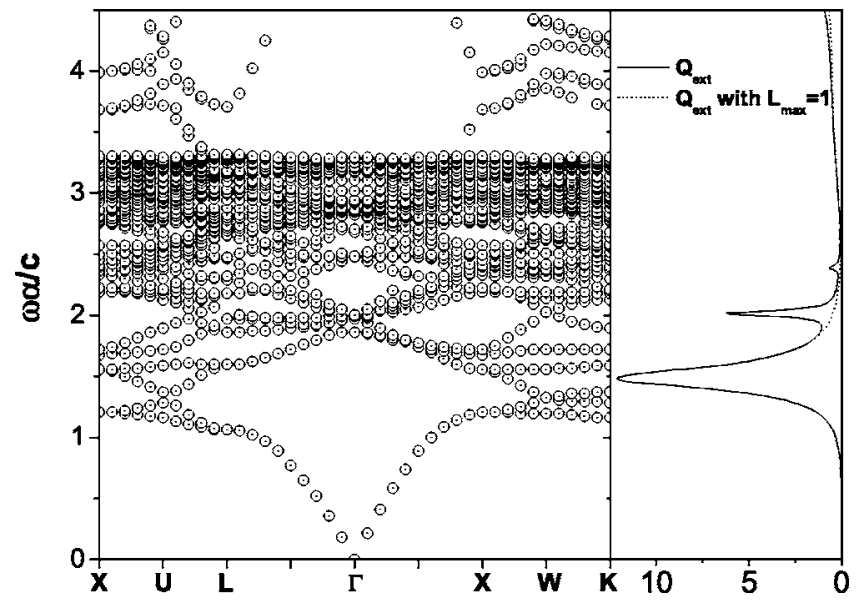

FIG. 6. Photonic band structure of the case $r_{\text {glass }}=0.44 \alpha$ of photonic crystal as described in Fig. 5 with $L_{\max }=7$. The right panel shows the extinction cross-section efficiency of a single particle.

gap of the crystal as shown in Fig. 8. Normally, a very small Ag sphere should have its Fröhlich surface mode at a very high frequency $(3.5 \mathrm{eV})$, but due to finite size effect, it is now shifted downwards in frequency to about $2 \mathrm{eV}$. Therefore, for the whole series of photonic crystal of Ag-coated glass spheres, the first photonic gap (a directional gap) is indeed due to the dipolar resonance of a single particle. It is known that the first photonic band gap of the FCC structure is very robust against disorder. This is because the gap is derived from resonance.

We now consider the simple cubic structure. For a simple cubic structure of Ag-coated glass particles with high enough filling ratio, the first photonic band gap is already an absolute gap. ${ }^{1}$ Figure 9 shows the transmittance for normal incidence on the (110) surface of a series of such photonic crystals with the filling ratio fixed at 0.435 , the outer radius of the coated particles fixed at $117.5 \mathrm{~nm}(0.47 \alpha, \alpha=250 \mathrm{~nm}$ is the lattice constant) and the glass core radius is again varied so that we can go from a pure glass sphere to a pure $\mathrm{Ag}$ sphere. The polarization of the E-field is aligned with the [100] direction.

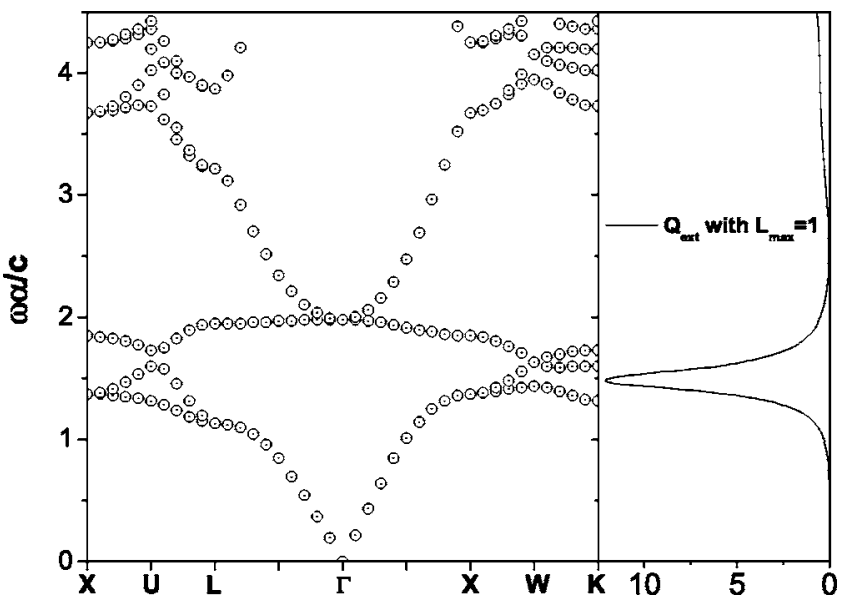

FIG. 7. Photonic band structure of the case $r_{\text {glass }}=0.44 \alpha$ of photonic crystal as described in Fig. 5 with $L_{\max }=1$. The right panel shows the extinction cross-section efficiency of a single particle. 


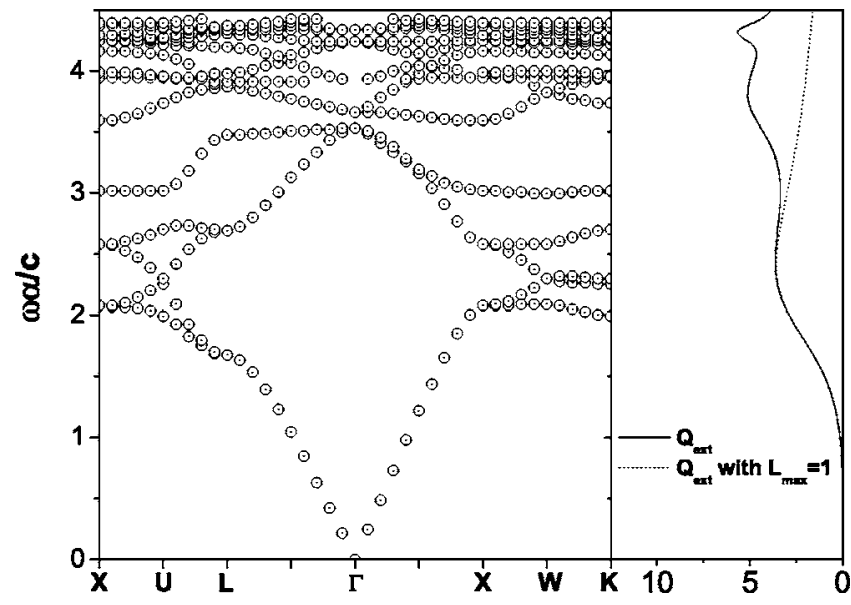

FIG. 8. Photonic band structure of photonic crystal as described in Fig. 5 in the case of pure $\mathrm{Ag}$ particles with $L_{\max }=7$. The right panel shows the extinction cross-section efficiency of a single particle.

With this polarization, the transmission spectra are probing the directional gap for the lower (slower velocity) branch of the dispersions along the [110] direction. For the SC structure, we note that the absolute gap is much smaller than the directional gap. By examining the exact band structures, the absolute gap only appears when the glass core radius is smaller than $0.44 \alpha$. For this whole series of photonic crystals, the first peak of the extinction cross-section efficiency of a single sphere correctly predicts the position of the resonance gap. The band structure for the case of $r_{\text {glass }}=0.43 a$ is shown in Fig. 10. By comparing with the band structure only up to the dipole term (in which the gap is closed at R), and then increasing the $L_{\max }$ step by step, we conclude that the dipole term opens a directional gap at the resonance frequency and the quadruple term $(L=2)$ is responsible for the further gap opening at the $\mathrm{R}$ point to get an absolute gap.

Since the dipolar resonance of a single metal particle opens a gap at that particular frequency, this can be useful in the design of photonic band gap materials. Here, we provide a map of the resonance condition (defined as the frequency of maximum polarizability) of metal-coated dielectric particle in Fig. 11. We note that the slope of the curves for different ratios between the radii of glass core and Ag coating is nearly zero at the regime of very small outer radius. It is because the resonance condition does not depend on the size of particle at the quasi-static limit. For example, the resonance condition of a small metallic particle at quasistatic limit is always given by its permittivity being minus two. On the other hand, as the outer radius of the particle increases, all the curves converge to a straight line passing through the origin corresponding to the case of a homogeneous perfect conducting particle. This resonance photonic gap should be more robust than the photonic gap arising from Bragg scattering under disorder since it is due to the resonance of a single particle, the Fröhlich surface mode.

To summarize, we have examined the optical properties of a photonic crystal composing of Ag-coated glass spheres. The origin of the first photonic band gap is due to the single sphere dipolar resonance, which is the Fröhlich surface mode

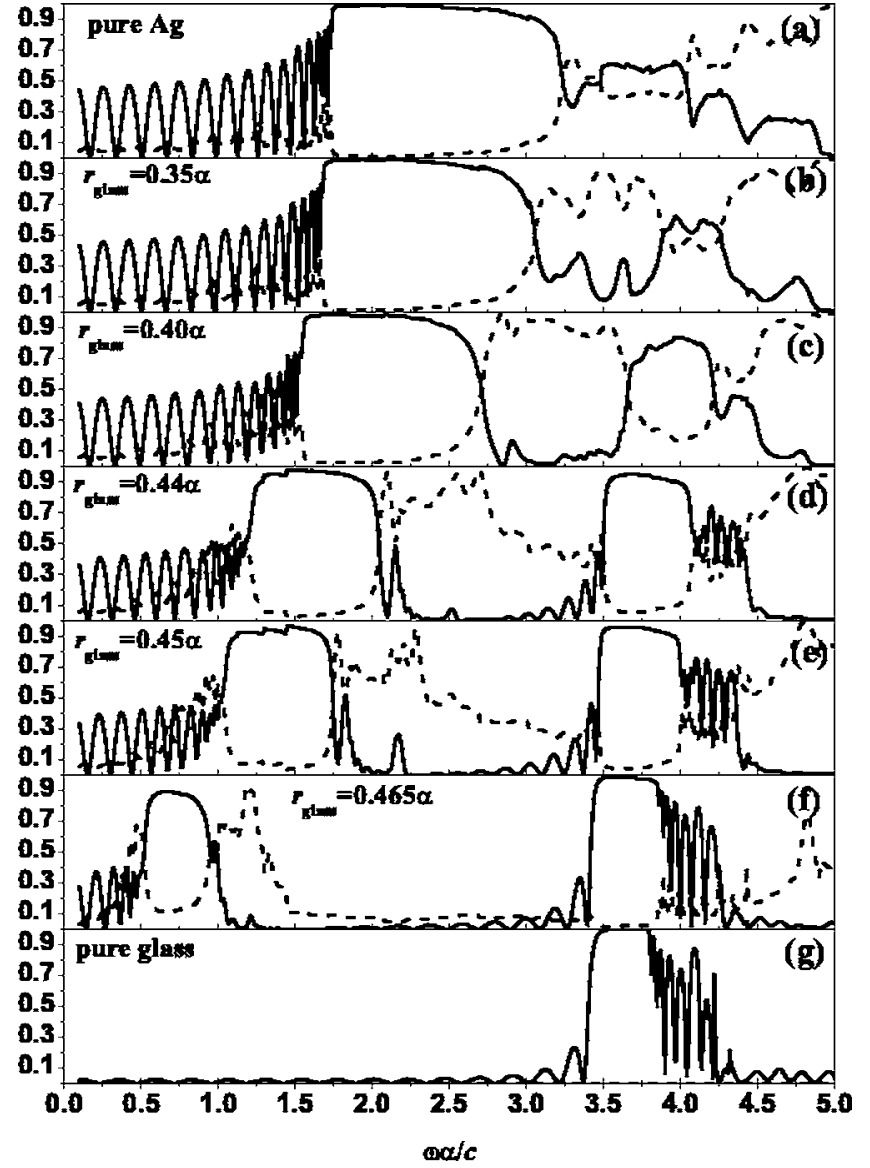

FIG. 9. Reflection spectrum (solid line) for normal incidence with E-field polarized along the [100] direction on 16 layers of SC (110) surface consisting of Ag-coated glass spherical particles at a volume filling ratio of 0.435 . The outer radius of the coated particle is fixed at $117.5 \mathrm{~nm}\left(r_{\mathrm{Ag}}=0.47 \alpha\right)$. The dashed lines show the corresponding absorbance.

in our case. However, it should be pointed out that this picture and the formulas derived in this work are also applicable to other kinds of single particle resonance in the low frequency regime. For metallic shells or metallic particles in nano-scale in which the classical bulk description of the metal fails, our formulation is still applicable by first calculating the dipolar response using nonlocal theory. The band structure in the whole Brillouin zone can be obtained by an extended effective medium theory we introduced. The resonance picture is generally a good approximation so that it can be a guidance principle in the design of metallodielectric photonic crystal. Photonic band gaps due to resonance are expected to be more robust than those arising from Bragg scattering.

\section{ACKNOWLEDGMENT}

This work is supported by RGC Hong Kong through Grant No. 600403.

\section{APPENDIX A: EFFECTIVE PERMITTIVITY OF A COATED PARTICLE}

In the low frequency regime where only the dipolar response is significant, a particle of isotropic electric polariz- 


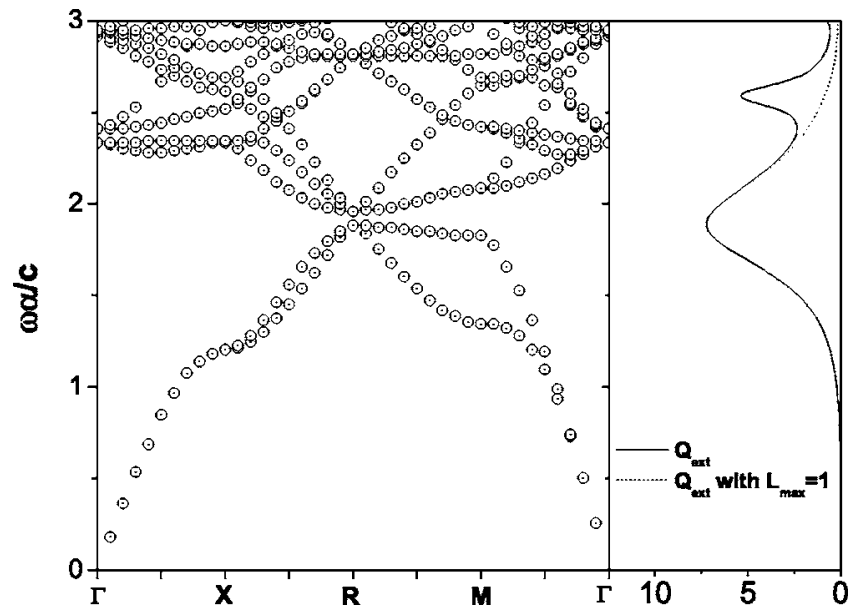

FIG. 10. Photonic band structure of photonic crystal as described in Fig. 9 in the case of $r_{\text {glass }}=0.43 \alpha$. The right panel shows the extinction cross-section efficiency of a single particle.

ability $\epsilon_{0} \alpha(\omega)$ can be effectively replaced by a homogeneous spherical particle of permittivity $\epsilon_{s}(\omega)$ of an arbitrarily chosen volume $\Omega_{s}$ (which should be small compared to the wavelength). By expanding the polarizability of the effective homogeneous particle in a Taylor series of frequency and taking only the leading order terms in its real and imaginary parts, the effective permittivity of the particle can be obtained from

$$
\frac{\epsilon_{s}+2}{\epsilon_{s}-1}=3 \Omega_{s}\left(\frac{1}{\alpha}+\frac{i k_{0}^{3}}{6 \pi}\right) .
$$

Equation (A1) is the basic equation to obtain the effective permittivity of the particle. Note that $\epsilon_{s}$ is purely real for a nonabsorbing particle from this equation. Suppose we are dealing with a coated spherical particle at the quasi-static limit (i.e., the local wavelength in each layer of the particle is much bigger than the radius of the particle), the polarizability of the particle does not need to be explicitly calculated using the spherical Bessel functions. We choose the volume of the effective homogeneous particle to be the same as the one of the original coated particle, Eq. (A1) can be further simplified to become

$$
\frac{\epsilon_{s}-\epsilon_{2}}{\epsilon_{s}+2 \epsilon_{2}}=\left(\frac{r_{1}}{r_{2}}\right)^{3} \frac{\epsilon_{1}-\epsilon_{2}}{\epsilon_{1}+2 \epsilon_{2}},
$$

where $r_{1} / r_{2}$ is the radius of the core/coating and $\epsilon_{1} / \epsilon_{2}$ is the permittivity of the core/coating. Equation (A2) has the same form as the Maxwell-Garnett formula. For more than one layer of coating, the formula can be first employed to replace the two innermost layers and this process can be iteratively carried out until the whole particle is effectively homogeneous. In the quasi-static limit, the resonance condition becomes independent on the size of the particle.

\section{APPENDIX B: DISPERSION OF A DIPOLAR CRYSTAL}

For a lattice of dipolar units in vacuum, let there be a Bloch state of wave vector $\boldsymbol{\kappa}$ (meaningful only in the first

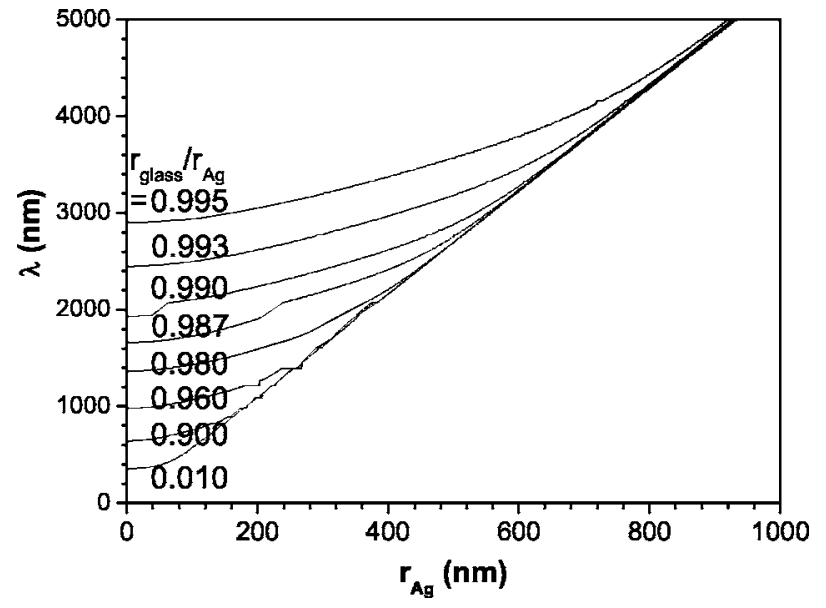

FIG. 11. Resonance condition for a Ag coated glass particle for different values of outer radius of the particle. $r_{\text {glass }} / r_{\mathrm{Ag}}$ is the inner/ outer radius of the particle. (The "wiggles" at $r_{\text {glass }} / r_{\mathrm{Ag}}=0.960$ is due to the noise in the $\mathrm{Ag}$ experimental data.)

Brillouin zone) so that we can write the dipoles as

$$
\mathbf{p}(\mathbf{R})=\mathbf{p}_{0} e^{i \boldsymbol{\kappa} \cdot \mathbf{R}},
$$

where $\mathbf{R}$ is a real space lattice vector. Then the local field at the origin site can be expressed as the macroscopic field $\langle\mathbf{E}\rangle$ minus the near field by continuum dipoles and add back the near field by discrete dipoles:

$$
\epsilon_{0} \mathbf{E}_{\text {local }}=\epsilon_{0}\langle\mathbf{E}\rangle+\frac{1}{\Omega_{a}} F\left[k_{0}^{2} \stackrel{\leftrightarrow}{G}(\mathbf{r})\right](\boldsymbol{\kappa}) \cdot \mathbf{p}_{0},
$$

where the macroscopic field is defined as the filtered version of the corresponding microscopic quantity such that only the spatial frequency components within the first Brillouin zone is retained. In the formula (B2), the Green's tensor for the background vacuum is given by

$$
\stackrel{\leftrightarrow}{G}(\mathbf{r})=\left(\overleftrightarrow{I}+\frac{1}{k_{0}^{2}} \nabla \nabla\right) \frac{e^{i k_{0} r}}{4 \pi r},
$$

where $\stackrel{\leftrightarrow}{I}$ is the identity tensor and $k_{0}$ is the wave number in vacuum. $\Omega_{a}$ is the volume of a single lattice site and the transform $F$ is defined by

$$
F[f(\mathbf{r})](\boldsymbol{\kappa})=\lim _{R_{0} \rightarrow \infty} \Omega_{a} \sum_{0<|\mathbf{R}|<R_{0}} f(\mathbf{R}) e^{i \boldsymbol{\kappa} \cdot \mathbf{R}}-\int_{0}^{R_{0}} d r^{3} f(\mathbf{r}) e^{i \boldsymbol{\kappa} \cdot \mathbf{r}}
$$

In the low frequency regime, we have the wavelength in vacuum much larger than the lattice constant $(a)$. There exists a finite value for $R_{0}$, the radius of the Lorentz sphere, to have the formula (B4) convergent. The radiation to the origin from the dipoles within the Lorentz sphere can be well approximated by the static propagator so that we can expand the Green's tensor within the Lorentz sphere in Taylor series of frequency and Eq. (B2) can be well approximated (up to the third order of frequency) in low frequency regime by 


$$
\begin{aligned}
\epsilon_{0} \mathbf{E}_{\text {local }}= & \epsilon_{0}\langle\mathbf{E}\rangle+\frac{1}{3} \frac{\mathbf{p}_{0}}{\Omega_{a}}+F\left[\frac{3 \hat{r} \hat{r}-\stackrel{\leftrightarrow}{I}}{4 \pi r^{3}}\right] \cdot \frac{\mathbf{p}_{0}}{\Omega_{a}} \\
& +k_{0}^{2} F\left[\frac{\stackrel{\leftrightarrow}{I}+\hat{r} \hat{r}}{8 \pi r}\right] \cdot \frac{\mathbf{p}_{0}}{\Omega_{a}}-\frac{i k_{0}^{3}}{6 \pi} \mathbf{p}_{0} .
\end{aligned}
$$

On the other hand, the single particle response can be expressed in terms of the frequency-dependent polarizability $\epsilon_{0} \alpha(\omega)$ by

$$
\mathbf{p}_{0}=\epsilon_{0} \alpha(\omega) \mathbf{E}_{\text {local }} \text {. }
$$

By combining Eqs. (B5) and (B6) together with the definition of $\overleftrightarrow{\epsilon}_{\text {eff }}$ from the constitutive relationship

$$
\langle\mathbf{P}\rangle=\frac{1}{\Omega_{a}} \mathbf{p}_{0} e^{i \boldsymbol{\kappa} \cdot \mathbf{r}}=\epsilon_{0}(\stackrel{\leftrightarrow}{\epsilon}(\boldsymbol{\kappa})-\stackrel{\leftrightarrow}{I}) \cdot\langle\mathbf{E}\rangle,
$$

and also the definition of $\epsilon_{s}$ in Appendix A, we obtain the wave-vector dependent permittivity given by

$$
\frac{\overleftrightarrow{\epsilon}_{\mathrm{eff}}(\boldsymbol{\kappa})+2 \stackrel{\leftrightarrow}{I}}{\overleftrightarrow{\epsilon}_{\mathrm{eff}}(\boldsymbol{\kappa})-\stackrel{\leftrightarrow}{I}}+3 F\left[\frac{3 \hat{r} \hat{r}-\stackrel{\leftrightarrow}{I}}{4 \pi r^{3}}\right]+3 k_{0}^{2} F\left[\frac{\overleftrightarrow{I}+\hat{r} \hat{r}}{8 \pi r}\right]=\frac{1}{f} \frac{\epsilon_{s}+2}{\epsilon_{s}-1} \stackrel{\leftrightarrow}{I}
$$

with volume filling ratio $f=\Omega_{s} / \Omega_{a}$. By solving the Maxwell equations with the wave-vector dependent permittivity, the corresponding eigenvalue problem is

$$
\left(\overleftrightarrow{U}_{s}(\boldsymbol{\kappa})+\frac{k_{0}^{2}}{\kappa^{2}-k_{0}^{2}}(\stackrel{\leftrightarrow}{I}-\hat{\kappa} \hat{\kappa})+k_{0}^{2} F\left[\frac{\stackrel{\leftrightarrow}{I} \hat{r} \hat{r}}{8 \pi r}\right]\right) \cdot \mathbf{p}_{0}=\frac{1}{3 f} \frac{\epsilon_{s}+2}{\epsilon_{s}-1} \mathbf{p}_{0},
$$

where the Fourier transformed dipole propagator is defined by $\overleftrightarrow{U}_{s}(\boldsymbol{\kappa})=\Omega_{a} \Sigma_{R>0}\left[(3 \hat{\mathbf{R}} \hat{\mathbf{R}}-\overleftrightarrow{I}) / 4 \pi R^{3}\right] e^{i \boldsymbol{\kappa} \cdot \mathbf{R}}$. On the left hand side of Eq. (B9), the second term is small for $\boldsymbol{\kappa}$ far away from the Brillouin zone center and it commutes with $\overleftrightarrow{U}_{s}(\boldsymbol{\kappa})$ for $\boldsymbol{\kappa}$ near the Brillouin zone center if the lattice is one of the cubic lattice type (FCC/BCC/SC). The third term is always small. Therefore, we use the eigenvectors of $\overleftrightarrow{U}_{s}(\boldsymbol{\kappa})$ as the unperturbed states and the dispersion can be further approximated by

$$
\frac{1}{3} \frac{\kappa^{2}-k_{0}^{2}}{k_{0}^{2} t_{i}(\boldsymbol{\kappa})+\left(\kappa^{2}-k_{0}^{2}\right)\left(\lambda_{i}(\boldsymbol{\kappa})+k_{0}^{2} \tau_{i}(\boldsymbol{\kappa})\right)}=f \frac{\epsilon_{s}-1}{\epsilon_{s}+2},
$$

where

$$
\begin{gathered}
\overleftrightarrow{U}_{s}(\boldsymbol{\kappa}) \cdot \hat{P}_{i}=\lambda_{i}(\boldsymbol{\kappa}) \hat{P}_{i} \quad \text { with } \hat{P}_{i} \cdot \hat{P}_{i}=1, i=1,2, \text { or } 3, \\
t_{i}(\boldsymbol{\kappa})=\hat{P}_{i} \cdot(\overleftrightarrow{I}-\hat{\kappa} \hat{\kappa}) \cdot \hat{P}_{i}, \\
\tau_{i}(\boldsymbol{\kappa})=\hat{P}_{i} \cdot F\left[\frac{\overleftrightarrow{I}+\hat{r} \hat{r}}{8 \pi r}\right] \cdot \hat{P}_{i} .
\end{gathered}
$$

By considering $\boldsymbol{\kappa}$ approaching the Brillouin zone center for a transverse mode $\left(t_{i}(\boldsymbol{\kappa})=1, \lambda_{i}(\boldsymbol{\kappa} \rightarrow 0)=1 / 3\right)$ and neglecting the dynamic-structure-correction term given by $k_{0}^{2} \tau_{i}(\mathbf{0})$, Eq. (B10) becomes

$$
\frac{\kappa^{2}-k_{0}^{2}}{\kappa^{2}+2 k_{0}^{2}}=f \frac{\epsilon_{s}-1}{\epsilon_{s}+2},
$$

which is the Maxwell-Garnett formula. Therefore, formula (B10) derived here is an extended version of MaxwellGarnett formula since it contains the dynamic-structurecorrection term and it covers the whole Brillouin zone. On the other hand, formula (B10) is compatible with the multiple-scattering approach except that it expands the band structure in the low frequency limit.
${ }^{1}$ W. Y. Zhang, X. Y. Lei, Z. L. Wang, D. G. Zheng, W. Y. Tam, C. T. Chan, and Ping Sheng, Phys. Rev. Lett. 84, 2853 (2000).

${ }^{2}$ V. Yannopapas, A. Modinos, and N. Stefanou, Opt. Quantum Electron. 34, 227 (2002).

${ }^{3}$ P. T. Miclea, A. S. Susha, Z. Liang, F. Caruso, C. M. Sotomayor Torres, and S. G. Romanov, Appl. Phys. Lett. 84, 3960 (2004).

${ }^{4}$ S. G. Romanov, A. S. Susha, C. M. Sotomayor Torres, Z. Liang, and F. Caruso, J. Appl. Phys. 97, 086103 (2005).

${ }^{5}$ P. Zhan, J. B. Liu, W. Dong, H. Dong, Z. Chen, Z. L. Wang, Y. Zhang, S. N. Zhu, and N. B. Ming, Appl. Phys. Lett. 86, 051108 (2005).

${ }^{6}$ S. J. Oldenburg, R. D. Averitt, S. L. Westcott, and N. J. Halas, Chem. Phys. Lett. 288, 243 (1998).

${ }^{7}$ Christina Graf and Alfons van Blaaderen, Langmuir 18, 524 (2002).

${ }^{8}$ P. Jiang, J. F. Bertone, and V. L. Colvin, Science 291, 453 (2001).

${ }^{9}$ S. L. Westcott, J. B. Jackson, C. Radloff, and N. J. Halas, Phys. Rev. B 66, 155431 (2002).

${ }^{10}$ R. D. Averitt, S. L. Westcott, and N. J. Halas, Phys. Rev. B 58,
R10203 (1998).

${ }^{11}$ S. J. Oldenburg, S. L. Westcott, R. D. Averitt, and N. J. Halas, J. Chem. Phys. 111, 4729 (1999).

${ }^{12}$ Traci R. Jensen, George C. Schatz, and Richard P. Van Duyne, J. Phys. Chem. B 103, 2394 (1999).

${ }^{13}$ F. Caruso, M. Spasova, V. Salgueirio-Maceira, and L. M. LizMarzn, Adv. Mater. (Weinheim, Ger.) 13, 1090 (2001); T. Cassagneau and F. Caruso, ibid. 14, 732 (2002); Z. J. Liang, A. Susha, and F. Caruso, Chem. Mater. 15, 3176 (2003).

${ }^{14}$ C. Graf and A. von Blaaderen, Langmuir 18, 524 (2002).

${ }^{15}$ E. Prodan, A. Lee, and P. Nordlander, Chem. Phys. Lett. 360, 325 (2002).

${ }^{16}$ J. B. Jackson, S. L. Westcott, L. R. Hirsch, J. L. West, and N. J. Halas, Appl. Phys. Lett. 82, 257 (2003).

${ }^{17}$ E. Prodan, C. Radloff, N. J. Halas, and P. Nordlander, Science 302, 419 (2003).

${ }^{18}$ T. V. Teperik, V. V. Popov, and F. J. García de Abajo, Phys. Rev. B 69, 155402 (2004).

${ }^{19}$ N. K. Grady, N. J. Halas, and P. Nordlander, Chem. Phys. Lett. 
399, 167 (2004).

${ }^{20}$ P. Nordlander, C. Oubre, E. Prodan, K. Li, and M. I. Stockman, Nano Lett. 4, 899 (2004).

${ }^{21}$ N. Stefanou, V. Yannopapas, and A. Modinos, Comput. Phys. Commun. 113, 49 (1998); A. Modinos, Physica A 141, 575 (1987).

${ }^{22}$ V. Yannopapas, A. Modinos, and N. Stefanou, Phys. Rev. B 60, 5359 (1999).

${ }^{23}$ J. E. Inglesfield, J. M. Pitarke, and R. Kemp, Phys. Rev. B 69,
233103 (2004).

${ }^{24}$ J. C. Maxwell Garnett, Philos. Trans. R. Soc. London, Ser. A 203, 385 (1904); 205, 237 (1906).

${ }^{25}$ C. F. Bohren and D. R. Huffman, Absorption and Scattering of Light by Small Particles (Wiley, New York, 1983).

${ }^{26}$ P. B. Johnson and R. W. Christy, Phys. Rev. B 6, 4370 (1972). Note that the data below the lowest frequency of experimental data is extrapolated using a Drude model.

${ }^{27}$ A. Liebsch and B. N. J. Persson, J. Phys. C 16, 5375 (1983). 